\title{
The need for a late follow-up cardiac magnetic resonance imaging to diagnose hypertrophic cardiomyopathy in patients with takotsubo syndrome
}

\author{
John E. Madias ${ }^{1,2}$ \\ Received: 11 December 2019 / Accepted: 28 December 2019 / Published online: 14 March 2020 \\ C) Springer Nature B.V. 2020
}

\section{To the Editor}

I enjoyed reading the report by Yalcinkaya et al. [1] in the Journal, about the patient with concomitant hypertrophic cardiomyopathy (HCM) and takotsubo syndrome (TTS). This is not the first case of a patient with TTS who has a comorbid HCM, as the authors indicated. Indeed, TTS occurs in patients with coronary artery disease, acute coronary syndromes, coronary dissection, HCM, and a large array of other cardiovascular and systemic pathologies. The patient was found to have "markedly thickened septum and obstruction at rest in the outflow tract, with a peak velocity of $323 \mathrm{~cm} / \mathrm{s}$ and an estimated peak gradient of $42 \mathrm{mmHg}$ indicating HCM" [1], although such phenotype is seen transiently in patients with TTS, albeit with apical ballooning, not encountered in this patient on echocardiography. The results of the cardiac magnetic resonance (CMR) performed in the subacute phase, and depicted in Fig. 1, of the paper under consideration [1], are very convincing in this patient case, that one deals with TTS and concomitant HCM. However, the edema of various myocardial territories in TTS produce an appearance of transient hypertrophy (i.e., pseudohypertrophy) indistinguishable from the one encountered in HCM [2], it is advisable that a delayed CMR follow-up study be implemented. Although all kinds of myocardial distributions are encountered in TTS, it is unlikely that the apical/septal involvement in the present case was due to TTS, and most probably it was due to apical TTS with concomitant HCM of the septal hypertrophy type with a intraventricular pressure gradient, as the authors suggested [1]. It is useful for the clinician and investigator to be mindful of the pseudohypertrophy occasionally encountered in TTS [2].

Funding No funding was received for this work.

\section{References}

1. Yalcinkaya D, Yarlioglues M, Yigit H, Duran M (2019) Murat SN Concomitant hypertrophic and takotsubo cardiomyopathy; cardiac magnetic resonance with parametric mapping findings after acute phase. Int J Cardiovasc Imaging 1:11. https://doi.org/10.1007/ s10554-019-01736-0

2. Madias JE (2016) Echocardiography-detected apical pseudohypertrophy and takotsubo syndrome. Int J Cardiol 220:279

Publisher's Note Springer Nature remains neutral with regard to jurisdictional claims in published maps and institutional affiliations.

This article refers to the article online at: Int J Cardiovasc Imaging. 2019 Nov 23. https://doi.org/10.1007/s10554-019-01736 -0 . [Epub ahead of print]

John E. Madias

madiasj@nychhc.org

1 Icahn School of Medicine At Mount Sinai, New York, NY, USA

2 Division of Cardiology, Elmhurst Hospital Center, 79-01

Broadway, Elmhurst, NY 11373, USA 